belief that industry suffers an understandable lack of knowledge about these things that the Department of Scientific and Industrial Research has attempted to provide an information channel from the academic producer of research results to the potential user in industry*.

* Department of Scientific and Industrial Research. Problems of Progress in Industry, No. 12: Human Sciences Aid to Industry. Pp. iv +27. (London: H.M.S.O., 1961.) 28.
Human Sciences Aid to Industry, one of the popular series Problems of Progress in Industry, discusses some of the main lines of research in the human sciences. Sufficient information is given from studies sponsored either wholly or partly by the Department of Scientific and Industrial Research to stimulate responsible people to take a more active interest in the subject.

\title{
CHEMOTHERAPY OF MALARIA
}

T HE importance of chemotherapy in the worldwide campaign for the eradication of malaria has been rapidly increasing. The clinician treating a limited number of malaria cases has at his disposal a complete series of effective drugs for treatment of all stages of the disease; but the malariologist dealing with a sick community, often in a region with limited public health services, may find socio-economic conditions a serious obstacle to making the fullest use of existing drugs or to using them at their full effectiveness for the purpose of malaria eradication.

A report*, prepared by the World Health Organization, considers, from the point of view of the malariologist, two broad questions: (1) What is the best use of antimalarial drugs ? (2) What new drugs are needed ? Some 16,000 antimalarial compounds have been studied and tested for antimalarial activity in recent years. The dozen or so which have survived this intensive screening and been found useful against human malaria are here given detailed consideration: the 4-aminoquinolines such as chloroquine, amodiquine, and their analogues; the 8-aminoquinolines

- World Health Organization. Technical Report Series, No. 226: Chemotherapy of Malaria - Report of a Technical Meeting. Pp. 92 . (Geneva: World Health Organization; London: H.M.S.o., 1961.) 3 Swiss francs; $58 . ; 1$ dollar. (pamaquine, primaquine, etc.); the pyrimidines and biguanides (pyrimethamine, proguanil and chlorproguanil); and a few others such as the older drug mepacrine. The usually recommended dosages are reviewed together with results achieved in mass drug administration.

Drug combinations and associations with an additive to counteract the shortcomings of certain drugs used singly are discussed with other aspects of malaria chemotherapy including the various ways of increasing the duration of action of the antimalarials -of special importance because of the difficulty of persuading entire populations to take drugs regularly and frequently for long periods; the principles of planning and organizing field trials of antimalarials; the problem of drug resistance, and possible ways of preventing or overcoming it; the practical problems of mass drug administration; and the use of medicated salt, a promising method of mass drug administration with which considerable experience has now been amassed in areas as diverse as Brazil, Cambodia, Ghana, Netherlands, New Guinea and British Guiana. An annex reviews variations in the modalities of drug administration in antimalaria programmes throughout the world.

\section{STAPHYLOCOCCAL CARRIAGE}

$\mathrm{T}$ THE carriage of staphylococei in sites such as the nose and throat, without the production of local or general disease, has become of increasing importance in recent years in relation to problems of crossinfection in hospitals and, to a lesser degree, among the population at large. The term 'carriage-rate' has never been accurately determined, however, and there is no general agreement regarding the sites to be examined, and even criteria of pathogenesis remain unsettled. An attempt at a quantitative survey of observations made from 1937 to 1959 in a number of countries-mainly in the British Commonwealth, Scandinavia and the United States-and based on material from many sites has recently been reported by E. Munch-Petersen. His findings have been sum. marized in a recent issue of WHO Chronicle $(15$, No. 11 ; November, 1961).

The carriage-rates in the general population and in hospitalized patients fluctuated very considerably from year to year and exhibited no obvious similarity in trend. A striking feature was the precipitate fall in rate among the hospital population during 1949, and possibly the outcome of the intensive use of antibiotics; but this fall left no impression on the subsequent trend, and the over-all hospital carriage-rate remained much the same. Nasal carriage-rates, taken alone, ranged widely: in the general population from 21.5 per cent (1953) to $49 \cdot 2$ per cent (1949); in hospital inmates from 32 per cent (1949) to $59 \cdot 2$ per cent (1959), again without any obvious pattern over the whole period. It is difficult to see why there should be such marked variations in carriage-rates in the same population from year to year, or why widely differing figures should be reported from different countries. The total rates for the general population ranged from a minimum of 13.6 per cent in some southern European countries to a maximum of 58 per cent in Germany; for hospital staffs and patients national rates varied between 28.1 per cent and 55.7 per cent. It is rare for two adjacent hospitals to have the same rate and there may even be differences between wards in the same institution.

The basis for these wide discrepancies may lie as much in variations of laboratory technique as in true differences of incidence. The methods of sampling, the sites sampled (the nose and skin of the hand are most generally used), the methods of culture and of performing coagulase and hæmolysin tests, all are subject to enough individual variation to cast doubt on the comparability of observations from different sources. At the moment it is only possible to reach some approximation to the true state of affairs. Not until there is a generally accepted method for determining staphylococcal carriage-rates, and generally accepted criteria of pathogenesis in staphylococci are available, will it be possible to 